\title{
Invasion percolation on a tree and queueing models
}

\author{
A. Gabrielli and G. Caldarelli \\ Dipartimento di Fisica, Centre SMC, INFM-CNR, Università di Roma "Sapienza," Piazzale A. Moro 2, 00185 Rome, Italy \\ and ISC, CNR, Via dei Taurini 19, 00185 Rome, Italy \\ (Received 9 July 2008; revised manuscript received 3 February 2009; published 22 April 2009)
}

\begin{abstract}
We study the properties of the Barabási model of queuing [A.-L. Barabási, Nature (London) 435, 207 (2005); J. G. Oliveira and A.-L. Barabási, Nature (London) 437, 1251 (2005)] in the hypothesis that the number of tasks grows with time steadily. Our analytical approach is based on two ingredients. First we map exactly this model into an invasion percolation dynamics on a Cayley tree. Second we use the theory of biased random walks. In this way we obtain the following results: the stationary-state dynamics is a sequence of causally and geometrically connected bursts of execution activities with scale-invariant size distribution. We recover the correct waiting-time distribution $P_{W}(\tau) \sim \tau^{-3 / 2}$ at the stationary state (as observed in different realistic data). Finally we describe quantitatively the dynamics out of the stationary state quantifying the power-law slow approach to stationarity both in single dynamical realization and in average. These results can be generalized to the case of a stochastic increase in the queue length in time with limited fluctuations. As a limit case we recover the situation in which the queue length fluctuates around a constant average value.
\end{abstract}

DOI: 10.1103/PhysRevE.79.041133 PACS number(s): 02.50.Le, 89.75.Da, 05.45.Tp, 89.65.Ef

\section{INTRODUCTION}

Queuing theory [1-3] and the study of task processing are theoretical problems that find application in a wide range of situations from human dynamics [4] to computer resource distribution [5]. Many of the traditional models introduced to explain that real queuing behaviors are based on the hypothesis of small or zero correlations for the execution of different tasks. This leads to exponential waiting-time distributions (WTDs) $P_{W}(\tau) \sim \exp \left(-\tau / \tau_{0}\right)$ for the execution of a generic task in the queue. This is the case of Poissonian processes, where the tasks are randomly chosen and executed with a constant rate. However, it has been recently noticed that for many human activities we have a large heterogeneity in waiting times. Long periods of quiescence between periods of high activity have been observed in real data [6]. In particular, this is the case of web browsing, electronic mail communications, and ordinary mail correspondence [7] for which a power-law decaying WTD has been observed. Much attention has therefore been paid during the last years to prioritydriven queuing models, i.e., models where each task is characterized by a random priority index. This index allows to reproduce these behaviors and these models generate powerlaw WTD $P_{W}(\tau) \sim \tau^{-\alpha}$ for the execution of the tasks at the stationary state.

Among these there is the Cobham [8] model of queuing where tasks enter the queue at rate $\lambda$, following an exponential arrival time distribution. The service time of each task follows an exponential distribution where tasks are executed at rate $\mu$. Each task is assigned a discrete priority parameter $x=1,2, \ldots, r$. If $p=1$ the highest priority item is always chosen for execution. In this hypothesis, Cobham derived the average waiting time for an item with priority $x$. Many variations in the model have been introduced but most of the work focused on the case when there are two priorities in the system $(r=2)$ and within each priority class items are executed on a first-come-first-serve fashion. This model is motivated by processes taking place in computer and industrial envi- ronments, where tasks are typically assigned only into two priority classes, high or low.

To consider a more general case we study in this paper a particular version of the Barabási queuing model (BQM) introduced in $[6,9]$. This model allows one to explain the stationary-state WTD observed. In our version of the BQM at each time step the task with highest random priority is always executed and replaced in the queue by a constant number $m \geq 2$ of new tasks with random priorities, i.e., the queue length grows linearly with time. This process can be mapped exactly onto an invasion percolation (IP) dynamics [10] on a Cayley tree [11] with a series of advantages. First, this allows one to characterize the task list dynamics through the WTD at the stationary state. Second, we can show that its general evolution is composed by a sequence of geometrically and causally connected burst of activities (task avalanches) with scale-invariant size distribution. Third, we can study the dynamics out of stationarity and show that the approach to it is very slow.

The hypothesis of a linearly growing queue length in time can look quite unrealistic, as many queuing services are thought to operate in situation of nearly constant queue length. However, the dynamics of receiving and answering to electronic mails and ordinary mail correspondence often constitute an example of strong deviation from the case of fixed queue length in time. This is evident in one particular case of study (the Darwin correspondence data set) shown in Fig. 1. In this case the number of letters sent by Charles Darwin (as a likely response to the fame of the scientist and the number of request received) oscillates strongly in time and increases steadily on average.

We then finally show that the main features of the model are retrieved when a randomly time-varying $m$ is considered with $\langle m\rangle \geq 1$ and finite $\left\langle m^{2}\right\rangle$. The lower limit of $m=1$ corresponds to the realistic case of a fluctuating queue length with constant average and finite fluctuations. 


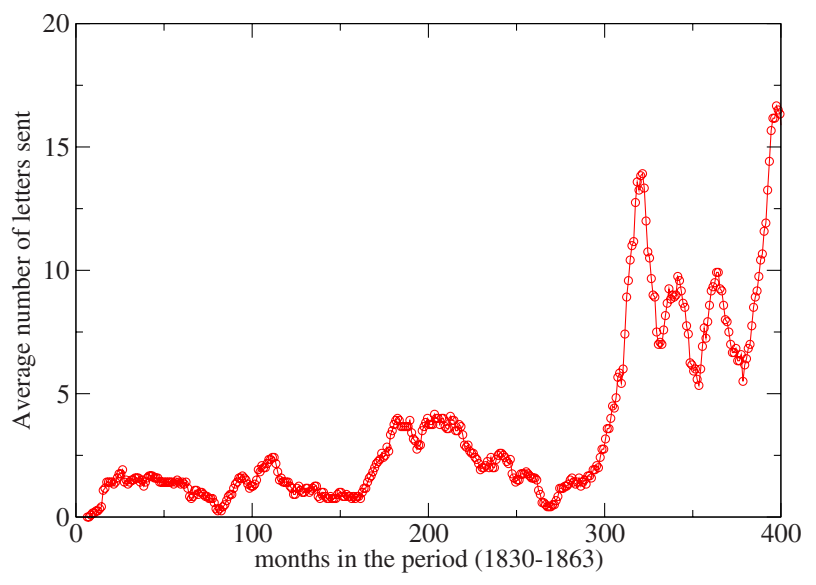

FIG. 1. (Color online) Plot of the number of letter sent per month by Charles Darwin. Data from the Darwin correspondence project at [12].

\section{MODEL}

In the general BQM [6] one starts with an initial list (i.e., queue) of $n_{0} \geq 2$ tasks. At every time step $t$ one of these tasks is executed and replaced by $m(t)$ other new tasks. When $m(t)=1$, the queue length is constant in time. The execution rule at each time step is given by fixing a random priority index $x_{i} \in[0,1]$ for each task in the queue and then executing with a probability $p \leq 1$ the task with the highest priority. With the complementary probability $(1-p)$ we instead execute a randomly chosen task. The related problem for general $0 \leq p \leq 1$ and $m=1$ has been analyzed and solved in $[13,14]$. In particular for $p$ smaller but close to 1 it has been shown in [13] that the system after a characteristic transient time $T(p)$ reaches a stationary state characterized by a WTD of the form

$$
P_{W}(\tau)=\frac{A(p)}{\tau} e^{-\tau / \tau_{0}(p)},
$$

such that $\tau_{0}(p) \rightarrow \infty$ and $A(p) \rightarrow 0$ for $p \rightarrow 1$ which makes the above expression meaningless in the value $p=1$. The stationary state for $p=1$ is trivial in the sense that at each time step the fresh new task just added to the queue is always executed. This is due to the fact that asymptotically the unchosen task staying in the queue has a priority $x=0$ with probability 1 , and consequently loses the competition with any new other task added to the queue. However in [14] it has been shown that $T(p)$ diverges for $p=1$ and that the trivial stationary state is reached only very slowly with a power-law approach in time. By mapping exactly the task list problem onto invasion percolation in $d=1$ and by using a probabilistic method called run time statistics (RTS) [15-17], the complete WTD $P_{W}\left(\tau, t_{0}\right)$ out of stationarity (which depends on both the entrance time $t_{0}$ of a task and on its waiting time $\tau$ ) has been found exactly. At fixed $t_{0}$ it decreases as $\tau^{-2}$ for $\tau$ $\gg t_{0}$ and is about $t_{0}^{-2}$ for $t_{0} \gg \tau$.

In the last period much attention has been devoted to the pure extremal case (i.e., with $p=1$ ) with a variable queue length for which the behavior of $P_{W}(\tau)$ differs strongly from the previous case. In [18] the case in which at each time step

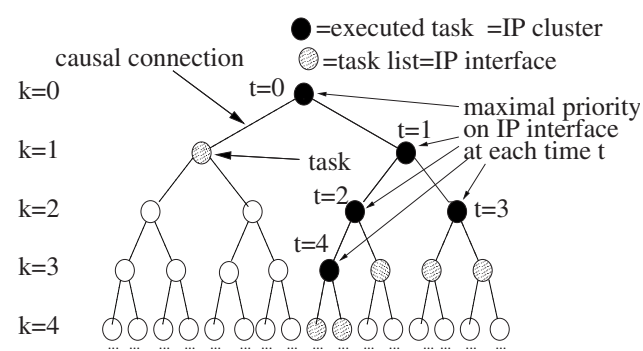

FIG. 2. Schematic of the first four steps of IP dynamics on a Cayley tree (with branching ratio $m=2$ ) into which the studied queuing model can be exactly mapped. The black sites indicates the growing set of occupied sites $\mathcal{C}_{t}$ (executed tasks) and the dashed ones the sites in the growth interface $\partial \mathcal{C}_{t}$. At each time step the site (task) with maximal fitness (priority) is occupied (executed) and replaced by $m$ new sites.

there is a probability $\mu \leq 1$ to execute the highest priority task has been studied, and at the same time a new task is added to the list with another probability $\nu \leq 1$. For $\mu=\nu$ $=1$ the above case of conserved queue length is recovered. If at least one between $\mu$ and $\nu$ is strictly smaller than 1 , the queue length instead varies stochastically in time and it is itself a stochastic variable performing a simple random walk with $(\mu \neq \nu)$ or without $(\mu=\nu)$ drift. Depending on $\mu>\nu$ or $\mu \leq \nu$ (with=sign only for $\nu<1$ ), the WTD $P_{W}(\tau)$ at the stationary state changes the asymptotic behavior. In the first case we have $P_{W}(\tau) \sim \tau^{-5 / 2} e^{-\tau / \tau_{0}}$, while in the second one we have $P_{W}(\tau) \sim \tau^{-3 / 2}$ with no upper cutoff for all the executed tasks with the following difference: for $\mu=\nu$ all tasks in the queue are asymptotically executed while for $\mu<\nu$ only tasks with $x_{i} \geq(1-\mu / \nu)$ are asymptotically executed as the mean list length grows linearly in time.

Our version of the model is strictly related to this last case as at each time step the task with highest priority is executed with probability $\mu=1$ and replaced in the queue by a constant number $m \geq 2$ of new tasks with random priorities. Therefore the queue length grows in time linearly. At every time step $(m-1)$ new tasks enter the queue. The motivation of this study relies on the fact that this model, which shares all the essential features with the previous one with $\mu<\nu$, can be exactly mapped into the IP dynamics on a Cayley tree (or Bethe lattice).

\section{INVASION PERCOLATION ON A CAYLEY TREE}

The use of IP in $d=1$ to describe queuing dynamics has already been presented [14] for the case of a strictly conserved queue length $(\mu=\nu=1$ in [18]). We extend here this approach to the more general case of queue length varying in time. Invasion percolation on a Cayley tree [11] is defined as follows (see Fig. 2): let us take a Cayley tree with branching ratio $m$ (or equivalently a Bethe lattice with coordination number $m+1)$. Initially only the top vertex site of the tree is occupied. A random number (fitness) $x \in[0,1]$, extracted from a uniform probability density function (PDF) $p(x)=1$, 
is assigned once and forever to each empty site independently of the others. ${ }^{1}$

At each time step the site of the growth interface $\partial \mathcal{C}_{t}$ with the highest fitness is occupied (i.e., grows). The interface $\partial \mathcal{C}_{t}$ is defined at each time $t$ as the set of empty sites connected by a first nearest-neighbor rule to the connected growing cluster $\mathcal{C}_{t}$ of occupied sites up to that time (see Fig. 2). Since for each occupied site other new $m$ sites enter the growth interface, the number of sites, respectively, in $\mathcal{C}_{t}$ and in $\partial \mathcal{C}_{t}$ grows in time, respectively, as $t$ and $\left|\partial \mathcal{C}_{t}\right|=m+(m-1) t$.

The exact mapping between IP and our queuing model is done by identifying sites with tasks, fitness with priority index, growth interface $\partial_{t}$ in IP with the task list (i.e., the queue), and finally the growing IP cluster $\mathcal{C}_{t}$ with the set of executed tasks up to time $t$. For our purposes we focus on the following features of the asymptotic stationary state of IP dynamics.

(1) Let us call $\phi(x, t) d x$ the distribution of fitnesses (priorities) of the sites (tasks) in $\partial_{t} \mathcal{C}$ (queue) at time $t$, i.e., the number of interface sites with fitness in $[x, x+d x]$ (also called normalized interface histogram). The asymptotic distribution $\phi_{s}(x)=\phi(x, t \rightarrow \infty)$ has the self-averaging stepfunction shape

$$
\phi_{s}(x)=p_{c}^{-1} \theta\left(p_{c}-x\right),
$$

where $p_{c}=(m-1) / m$ is the ordinary percolation threshold ${ }^{2}$ of the Cayley tree. This implies that (i) apart from a vanishing fraction of sites, all the interface sites have fitness $x<p_{c}$; (ii) since the number of sites in the stationary state is infinite, only those few sites with $x \geq p_{c}$ can grow at each time step. Indeed, at each time for the just occupied site (executed task) $m \geq 2$, new sites (new tasks) enter the interface (queue). This implies that a fraction $(m-1) / m$ of the sites entered the interface at any time will never be executed. Since the interface site with maximal fitness is always executed and the "fresh" interface sites have random fitness, asymptotically only and all the sites with $x \geq(m-1) / m$ are executed while the others stay forever in the interface.

(2) The cluster of occupied sites substantially coincides with the incipient percolating cluster of ordinary percolation (i.e., at occupation probability $p=p_{c}$ ).

(3) The stationary dynamics self-organizes into a sequence of spatially and causally connected critical avalanches of growths as defined in [19] with a scale-invariant size distribution (for a real case see Fig. 3). Due to the asymptotic shape (1) of the fitness distribution of the IP interface (task queue) $\mathcal{\partial} \mathcal{C}_{t}$, at very large time, when the number

\footnotetext{
${ }^{1}$ In the original physical interpretation IP [10] describes the quasistatic displacement in a porous medium of a fluid by another fluid having larger surface tension. In this picture sites or bonds of the lattice represent the throats of the porous medium and the site fitness gives the random capillarity of the throat.

${ }^{2}$ Note that in the usual version of ordinary percolation $p_{c}=1 / \mathrm{m}$. This different threshold is due to the fact that in our formulation of IP, we occupy at each step the site with the maximal fitness instead of the minimal one as usually done for IP. Clearly one dynamics is mapped into the other by simply substituting each $x$ with $(1-x)$. This explains the different value of the percolation threshold.
}

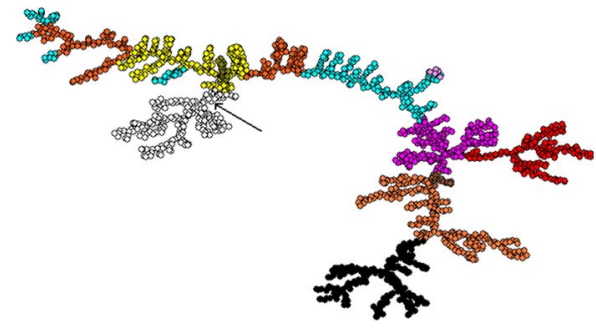

FIG. 3. (Color online) A plot of the avalanches of the executed task over a Cayley tree (rearranged). The arrow indicates the starting task and different gray levels refer to different avalanches. The plot has been done thanks to the PAJEK software.

of sites (tasks) in $\partial \mathcal{C}_{t}$ diverges, it is impossible that a site with fitness $x<p_{c}$ grows. This happens because at each time step there is at least a site with $x \geq p_{c}$ (see Fig. 4). Let us suppose that at a given time step in the stationary state a task with fitness $x \geq p_{c}$ grows. Due to the IP dynamical rules and Eq. (1), if at the next time step a site with fitness $y>x$ is occupied, this is a causally and geometrically connected event to the previous one. Indeed this site can only be entered, the growth interface, because of the growth of the site with fitness $x$ which was the maximal value on the interface at the previous time step. Therefore one can define a causally and geometrically connected $x$ avalanche of growth events initiated by the occupation of a site (avalanche initiator) with fitness $x \geq p_{c}$ as the sequence of growths of sites with fitness $y>x$ following the one of the initiator.

When a site with fitness $y \leq x$ (but clearly $y \geq p_{c}$ ) grows for the first time from the beginning of the $x$ avalanche, the $x$ avalanche stops. It is simple to realize [19] that this defini-

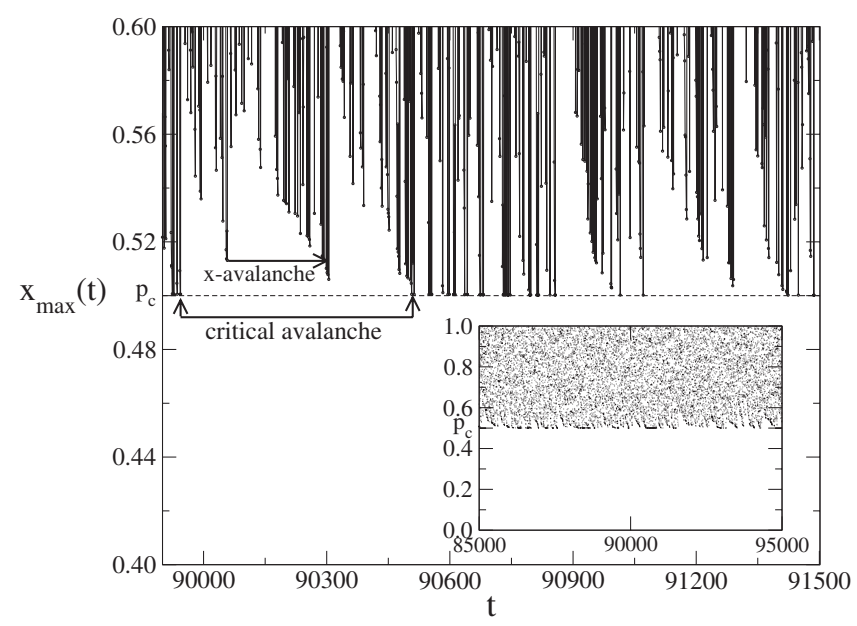

FIG. 4. Time sequence of the priority indexes of the selected tasks in one realization of the model with $m=2$ and time duration $10^{5}$. In the main plot a zoom of the sequence in a restricted window $\left(t \in[89900,91500]\right.$ and $\left.x \in\left[p_{c}-0.1, p_{c}+0.1\right]=[0.4,0.6]\right)$ is shown. We see that asymptotically only tasks with $x \geq p_{c}$ are executed and that task executions organize in hierarchical macroevents (avalanches). In particular we pointed out two examples of, respectively, a generic $x$ avalanche and of a critical avalanche whose initiators has $x=p_{c}$. In the inset the sequence of tasks priorities with all possible $x$ values $(x \in[0,1])$ and larger time window $t$ $\in[85000,95000]$ is shown. 


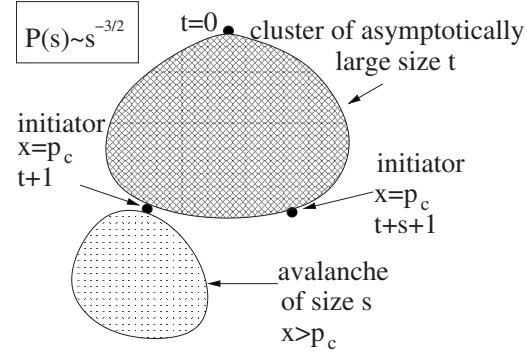

FIG. 5. Illustration of a causally and geometrically connected critical avalanche at the stationary state whose initiator has $x=p_{c}$. Such an avalanche is characterized by the causal connection consisting in the fact that all the growth belonging to it form a time sequence and have $x>p_{c}$. Its size distribution is $P(s) \sim s^{-3 / 2}$. This characterizes the stationary state of both IP on the tree and the task queue dynamics.

tion of the $x$ avalanche gives rise to a complex hierarchical structure of nested avalanches. ${ }^{3}$ In this hierarchy only $p_{c}$ avalanches or critical avalanches are completely geometrically disconnected (see Fig. 5). As shown below explicitly, the size distribution of the $x$ avalanches is

$$
P(s ; x) \sim s^{-\alpha} \exp \left[-\left(x-p_{c}\right)^{\sigma} s\right],
$$

where $\alpha=3 / 2$ and $\sigma=2$. Therefore for $x=p_{c}$ the distribution is a pure power law $P(s) \sim s^{-3 / 2}$ (see Fig. 6).

We now deduce Eq. (1) and the exponents $\alpha$ and $\sigma$ of the avalanche distribution in Eq. (2) in the stationary state. Let $n(x, t)$ be the number of sites (tasks) with fitness (priority) larger than $x$ in the interface (queue) after the $t$ th dynamical step. Since after the growth of one site $m$ new sites enter the interface, we have the following Markovian evolution for $n(x, t)>0$ :

$$
\begin{aligned}
n(x, t+1)= & n(x, t)+j-1 \\
& \text { with probability }\left(\begin{array}{c}
m \\
j
\end{array}\right) x^{m-j}(1-x)^{j},
\end{aligned}
$$

with $j=0,1, \ldots, m$. That is, $n(x, t)$ follows an ordinary random walk with independent steps which can take integer values from -1 to $m-1$. The average increment at each time step is $\overline{\delta n}(x)=[m(1-x)-1]=m\left(p_{c}-x\right)$ which is negative for $x>p_{c}$, exactly zero for $x=p_{c}$ (martingale property), and positive for $x<p_{c}$. In other words for $x=p_{c}$ the random walk performed by $n(x, t)$ has no drift, while for $x<p_{c}$ and $x$ $>p_{c}$ the drift is, respectively, positive and negative. This simple observation explains the origin of Eq. (1); the number of sites (tasks) with fitness larger than $x<p_{c}$ in average grows linearly in time as $\overline{\delta n}(x) \times t$ with fluctuations of order $t^{1 / 2}$. More precisely at sufficiently large $t$ we can apply the central limit theorem to say that $n(x, t)$ for $x<p_{c}$ is Gaussian distributed with mean value

\footnotetext{
${ }^{3}$ Note that Eq. (1) permits that, when a site with $x>p_{c}$ grows in the stationary state, at the next time step a site with $y \leq x$ geometrically connected to it grows. This is however not possible if $x=p_{c}$ since, as shown above, no site with $x<p_{c}$ can grow in the stationary state.
}

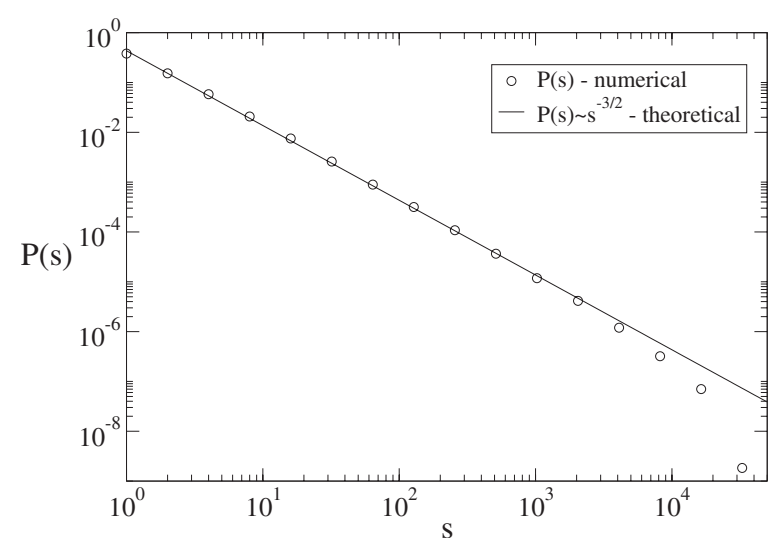

FIG. 6. Plot of critical avalanche size distribution for $m=2$. It is obtained by averaging $10^{3}$ realizations of the dynamics up to $t=5 \times 10^{4}$.

$$
\langle n(x, t)\rangle=\left\langle n_{0}(x)\right\rangle+m\left(p_{c}-x\right) t,
$$

where $n_{0}(x)$ is the initial number of interface sites ${ }^{4}$ with fitness larger than $x<p_{c}$, and variance

$$
\left\langle n^{2}(x, t)\right\rangle-\langle n(x, t)\rangle^{2}=\left\langle\Delta n_{0}^{2}(x)\right\rangle+\Delta^{2} t,
$$

with $\Delta^{2}=m(m-1)(1-x)^{2}-m\left(p_{c}-x\right)-m^{2}\left(p_{c}-x\right)^{2}$. Since the total number of interface sites grows as $m+(m-1) t$, we have that at large $t$ the fraction of interface sites whose fitness is larger than $x<p_{c}$ (i.e., the integrated fitness distribution $\psi(x, t)=\int_{x}^{1} d y \phi(y, t) \equiv n(x, t) /[(m-1) t]$ of the tasks in the queue) is also a Gaussian variable with mean given by

$$
\langle\psi(x, t)\rangle=\frac{\left\langle n_{0}(x)\right\rangle}{m+(m-1) t}+\frac{m\left(p_{c}-x\right) t}{m+(m-1) t}
$$

and variance

$$
\left\langle\psi^{2}(x, t)\right\rangle-\langle\psi(x, t)\rangle^{2}=\frac{\left\langle\Delta n_{0}^{2}(x)\right\rangle}{(m-1)^{2} t^{2}}+\frac{\Delta^{2}}{(m-1)^{2} t} .
$$

On the other hand since $\overline{\delta n}(x) \leq 0$ for $x \geq p_{c}$, interface sites with fitness $x \geq p_{c}$ give only a vanishing fraction to the fitness distribution at large times. All this leads exactly to Eq. (1) in the infinite time limit showing the self-averaging of this relation.

For what concerns the avalanche size distribution, we are interested in the stationary state. As aforementioned, due to Eq. (1), in this case only sites with $x \geq p_{c}$ can grow. Let us now interpret $t$ as the number of time steps elapsed from the growth of the initiator of the $x$ avalanche in the stationary state. Until the time step $t>0$ at which $n(x, t)=0$ for the first time, $n(x, t)$ can also be seen as the number of sites causally connected to the initiator in the sense explained above. Consequently, the duration $s$ of the $x$ avalanche is none other than the number of time steps after which $n_{s}\left(p_{c}\right)=0$ for the first time after the growth of the initiator. Let us set $x=p_{c}$. As seen above in this case the random walk performed by $n_{t}\left(p_{c}\right)$

\footnotetext{
${ }^{4}$ If, for instance, as in Fig. 2, the initial interface is constituted by $m$ sites with random fitness, we have $\left\langle n_{0}(x)\right\rangle=m(1-x)$ and $\left\langle\Delta n_{0}^{2}(x)\right\rangle=m(m-1)(1-x)^{2}$.
} 
has no drift, i.e., is unbiased. From the theory of random walks we know that in the unbiased case [20] the distribution of $s$ has the scale-invariant form $P(s) \equiv P\left(s ; p_{c}\right) \sim s^{-3 / 2}$. This means that in Eq. (2) $\alpha=3 / 2$. When instead $x>p_{c}$ we have $\overline{\delta n}(x)=m\left(p_{c}-x\right)<0$, and therefore the drift of the walk is constant toward the absorbing state $n(x, t)=0$. Therefore the average size of such an $x$ avalanche is $s_{0} \sim\left(x-p_{c}\right)^{-1}$. From Eq. (2) with $\alpha=3 / 2$, this implies that $\sigma=2$.

A percolation argument can also be used to find out the same exponents. Let us take an avalanche whose initiator has fitness $x \geq p_{c}$. The avalanche lasts exactly for a time interval equal to the number of sites with fitness larger than $x$ connected to it in the positive time direction. Therefore the avalanche size is distributed as the finite clusters in ordinary percolation on the same tree for the occupation probability $p=x[21]$, i.e., Eq. (2) with the given exponents.

Random walk and diffusion theory arguments also permit to evaluate the stationary-state WTD $P_{W}(\tau)$ for the tasks with $x \geq p_{c}$. We follow here a similar discussion to [18]. We can write the WTD as

$$
P_{w}(\tau)=\sum_{n=0}^{\infty} \int_{p_{c}}^{1} d x \widetilde{Q}(n, x) G(n, x, \tau),
$$

where $\widetilde{Q}(n, x)$ is the probability that at a generic time step at the stationary state, we have exactly $n$ tasks in the queue (i.e., sites on the IP interface) with priority larger than $x$ $\geq p_{c}$. The quantity $G(n, x, \tau)$ is instead the conditional probability that always at the stationary state, a certain task with priority $x \geq p_{c}$ added to the list at a time step when other $n$ tasks with priority larger than $x$ are present is executed after $\tau$ time steps.

Starting from Eq. (3), we can write the master equation for the probability $Q(n, x, t)$ that at time step $t$ of the dynamics, there are exactly $n$ tasks in the list with priority larger than $x$. With the aim of simplicity, let us write it for $m=2$ for which $p_{c}=1 / 2$. From Eq. (3) we can write for $n \geq 3$

$$
\begin{aligned}
Q(n, x, t+1)= & Q(n+1, x, t) x^{2}+Q(n, x, t) 2 x(1-x) \\
& +Q(n-1, x, t)(1-x)^{2},
\end{aligned}
$$

while for $n \leq 2$ we have

$$
\begin{gathered}
Q(2, x, t+1)=Q(3, x, t) x^{2}+Q(2, x, t) 2 x(1-x) \\
+Q(1, x, t)(1-x)^{2}+Q(0, x, t)(1-x)^{2}, \\
Q(1, x, t+1)=Q(2, x, t) x^{2}+Q(1, x, t) 2 x(1-x) \\
+Q(0, x, t) 2 x(1-x), \\
Q(0, x, t+1)=Q(1, x, t) x^{2}+Q(0, x, t) x^{2} .
\end{gathered}
$$

$\widetilde{Q}(n, x)$ is given by the stationary solution of the above equations. In order to find both $\widetilde{Q}(n, x)$ and $G(n, x, \tau)$ we can now proceed in a similar way to [18]. It is simple to show that the well-normalized stationary solution for $x \geq p_{c}=1 / 2$ of Eqs. (7) and (8) is

$$
\tilde{Q}(n, x)=\frac{2\left(x-p_{c}\right)}{x^{2}}\left[\frac{(1-x)^{2}}{x^{2}}\right]^{n-1} \text { for } n \geq 2,
$$

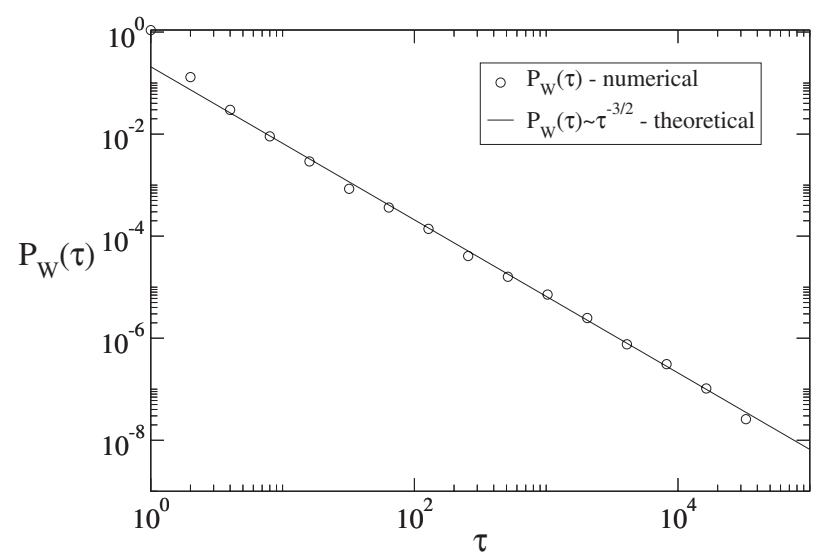

FIG. 7. Plot of the waiting-time distribution at the stationary state for $m=2$. It is obtained by averaging $10^{3}$ realizations of the dynamics up to $t=5 \times 10^{4}$.

$$
\tilde{Q}(1, x)=2 \frac{1-x^{2}}{x^{2}}\left(x-p_{c}\right), \quad \tilde{Q}(0, x)=2\left(x-p_{c}\right) .
$$

Note that for $x \rightarrow p_{c}^{-}$any $\tilde{Q}(n, x) \rightarrow 0$ with the ratio $\widetilde{Q}(n, x) / \widetilde{Q}(l, x) \rightarrow 1$ for any $n, l \geq 2$, i.e., the distribution of the number $n_{t \rightarrow \infty}\left(p_{c}\right)$ becomes practically uniform.

The quantity $G(n, x, \tau)$ can be found by Eq. (7) in complete analogy with $[18,22]$, leading both to the same correct scaling behavior $P_{W}(\tau) \sim \tau^{-3 / 2}$. We refer here to [18] as it is of simpler formulation than [22]. First of all we note that Eq. (7), in both the continuous time and $n=y$ approximation, becomes the diffusion equation

$$
\partial_{t} Q(y, x, t)=c(x) \partial_{y}^{2} Q(y, x, t)+d(x) \partial_{y} Q(y, x, t),
$$

with $c(x)=x^{2}$ and $d(x)=x^{2}-(1-x)^{2}$. Since we are considering $x \geq p_{c}=1 / 2$ we have $d(x) \geq 0$, i.e., there is a drift to the small $y$ (i.e., $n$ ) direction, as already pointed out by analyzing Eq. (3). The quantity $G(n, x, \tau)$ can be seen as the probability that at the stationary state, fixed $x$ and given $y=n$ at time $t=0$, one has $y=0$ for the first at time $t=\tau$. This implies that $[18,20]$

$$
G(n, x, t)=-\partial_{t}\left[\int_{0}^{\infty} d y Q(y, x, t)\right],
$$

where here $Q(y, x, t)$ is the solution of Eq. (10) with initial condition $Q(y, x, 0)=\delta(y-n)$. All this gives

$$
G(n, x, t)=\frac{n}{\sqrt{4 \pi c(x)} t} \exp \left\{-\frac{[n-d(x) t]^{2}}{4 c(x) t}\right\} .
$$

We now use this result and Eq. (9) in Eq. (6) to find $P_{W}(\tau)$. It is simple [18] to show that for large $\tau$ we have $P_{W}(\tau)$ $\sim \tau^{-3 / 2}$ (see Fig. 7).

In other words each task with $x \geq p_{c}$ has to wait a finite portion of the avalanche duration before being executed. Note that this scaling result, explicitly found for $m=2$, is completely independent of the integer branching factor $m$ $>1$. Using a different constant value of $m>2$ only changes 
the explicit expressions of $G(n, x, t)$ and $\widetilde{Q}(n, x)$ but not Eqs. (10) and (11) with $c(x) \geq 0$ and $d(x)$ changing sign at $x=p_{c}$ $=(m-1) / m$. This is sufficient to recover the scaling relation $P_{W}(\tau) \sim \tau^{-3 / 2}$. From Eq. (3) it is natural to expect to have the same result in the case in which at each time step $m$ is an independent random variable with mean $\langle m\rangle \geq 1$ and finite and positive variance. In particular the case of $\langle m\rangle=1$ refers to the situation of a constant queue length in average with finite but nonzero fluctuations. Indeed also in this case the number of tasks in the queue with priority larger than $x$ performs an ordinary random walk. The reason why both cases of constant $m \geq 2$ and random $m$ with $\langle m\rangle \geq 1$ and finite variance belong to the same universality class is the following. In both cases the number of tasks in the queue with priority larger than $x \geq p_{c}$ [where $p_{c}=(m-1) / m$ in the deterministic case and $\left.{ }^{5} p_{c}=(\langle m\rangle-1) /\langle m\rangle\right]$ performs the same type of ordinary random walk which is negatively biased for $x>p_{c}$ and unbiased for $x=p_{c}$. On the other hand tasks with $x<p_{c}$ are never executed in the stationary state (this is well pictorially shown by the mapping to invasion percolation on a tree). Therefore they are all governed by the same laws of first passage of ordinary random walks which leads to the scaling $P_{W}(\tau) \sim \tau^{-3 / 2}$. This explains also why our model shares the same statistical features with that in [18]. Note anyway that this is not true in the deterministic case of constant $m=1$ in which the number of tasks in the queue is constantly equal to two and no random walk is performed by it or any subset of it. Indeed in this case a different scaling is found $[13,14]$. In the case where $m$ is a random variable with $\left\langle m^{2}\right\rangle=+\infty$ we finally expect anomalous exponents for both $P(s)$ and $P_{W}(\tau)$ as the random walks expressed by Eq. (3) become Levy flights as shown in [23].

We now address the question of the velocity of the approach to stationarity in this model. Also this problem can be solved by the use of theoretical tools from random-walk theory and IP on a tree. First of all we have to distinguish the relaxation to stationarity in a single realization of the queue dynamics (which, as shown below, is dominated by random fluctuations) from the relaxation of characteristic quantities averaged over all dynamical realizations (which show a slow and smooth transition from the initial value to the stationary one). We start from the first one by summarizing the main results in literature about IP and comparing it with the equivalent results from random-walk theory. After that we study the relaxation of averaged quantities such as the fitness distribution in the queue averaged over all realizations, proposing a simple mean-field approach leading to right results and showing how slow the relaxation to the right stationary state is.

In [24] it is rigorously shown that (i) the IP cluster on a Cayley tree has in the infinite time limit a unique backbone. In terms of the task dynamics this means that there is a unique infinite chain of executed tasks which are causally connected in the IP sense above. (ii) The minimal priority of the executed tasks staying on the backbone beyond the $k$ th generation of the Cayley tree (see Fig. 2) is $p_{c}(1-Z / k)$ for

\footnotetext{
${ }^{5}$ In particular for the random $m$ case with $\langle m\rangle=1$ and finite variance, $p_{c}=0$.
}

large $k$ where $Z$ is an exponentially distributed random variable with unitary mean. In [11] it is instead rigorously shown that adapting the notation to our queuing problem, the probability that at time $t$ of a single dynamical realization a task with priority smaller than $\left(p_{c}-\epsilon\right)$ is executed, vanishes exponentially fast at large $t$ for $\epsilon>0$ but as $t^{-1 / 2}$ for $\epsilon \rightarrow 0^{+}$. This suggests that deviations from the stationary dynamics disappear as $t^{-1 / 2}$. This is consistent with Eq. (3). Indeed, as aforementioned, for $x<p_{c}$ we see from Eqs. (4) and (5) that relative fluctuations of $\psi(x, t)$ with respect to its mean value decrease ${ }^{6}$ at large $t$ as $t^{-1 / 2}$.

Let us now consider the same quantity averaged over all possible realizations of the dynamics. We have shown that the approach to the stationarity is now described by the behavior of $\langle\psi(x, t)\rangle$. For $x<p_{c}$ this is given by Eq. (4) which shows that deviations in this region from the stationary state [which is described by Eq. (1)] disappear as fast as $t^{-1}$, i.e., faster than in a single realization even though still very slowly. For $x>p_{c}$, as aforementioned, the average value and the variance of $n(x, t)$ do not increase with $t$ at large time, and therefore $\langle\psi(x, t)\rangle \rightarrow 0$ as $t^{-1}$ again. This shows that even washing out the stochastic fluctuations by an average over dynamical realizations, we have a transition to the stationary state from the initial one that is as slow as $t^{-1}$.

\section{CONCLUSIONS}

In conclusion we presented in this paper an analytical approach to the Barabási model of human dynamics with time-increasing queue length. We proposed a combined approach to the problem based on IP and random-walk theory. These methods allowed us to describe quantitatively two intuitive features of the queues. The first one is that some tasks seem to remain indefinitely in the queue before being processed. Second we recovered naturally the fact that in the real world execution of a task has often the effect of generating an avalanche of new tasks and that executed tasks wait in the queue a broadly distributed time before their execution. This approach has also allowed to study the approach of the dynamics to the stationary state and to show how slow it is. All these properties of the dynamics are characterized by temporal power laws typical of extremal dynamics in quenched disorder [15-17]. We considered the case in which for each executed task $m \geq 2$ new tasks are added to the queue. However, [as shown above by the random-walk methods used to describe the dynamics and in particular by Eq. (3)] all the main results related to both the stationary state and the approach to it hold also when $m$ is a stochastic variable $(m \geq 1)$ with finite variance. Indeed in this case the nature of the random walk is kept and the time scalings of the first passage probabilities and of the average value and variance of $n(x, t)$ are maintained. In particular this includes the case of fluctuating $m$ with mean value $\bar{m}=1$ which refers to the case of a fluctuating queue length with constant mean

\footnotetext{
${ }^{6}$ Since for $x>p_{c}$ the constant drift $\overline{\delta n}(x)<0, n(x, t)$, has at any $t$ mean and variance that are independent of $t$. Therefore fluctuation of $\psi(x, t)$ in this region decreases as $1 / t$.
} 
value (coinciding with the case $\mu=\nu<1$ in [18]). This is usually considered as the situation in technological services. However there are important cases, such as electronic mail- ing and ordinary mail correspondence, in which the description as a queue with length growing in time is more appropriate as shown from available data sets [12].
[1] D. R. Cox and W. L. Smith, Queues (Methuen, London, 1961).

[2] D. Gross and C. M. Harris, Fundamentals of Queueing Theory, 3rd ed. (Wiley, New York, 1998).

[3] L. Breuer and D. Baum, An Introduction to Queueing Theory and Matrix-Analytic Methods (Springer, New York, 2005).

[4] P. Reynolds, Call Center Staffing (The Call Center School, Tennessee, 2003).

[5] H. R. Anderson, Fixed Broadband Wireless System Design (Wiley, New York, 2003).

[6] A.-L. Barabási, Nature (London) 435, 207 (2005).

[7] A. Vazquez, J. G. Oliveira, Z. Dezso, K. I. Goh, I. Kondor, and A. L. Barabási, Phys. Rev. E 73, 036127 (2006).

[8] A. Cobham, J. Oper. Res. Soc. Am. 2, 70 (1954).

[9] J. G. Oliveira and A.-L. Barabási, Nature (London) 437, 1251 (2005).

[10] D. Wilkinson and J. F. Willemsen, J. Phys. A 16, 3365 (1983).

[11] B. Nickel and D. Wilkinson, Phys. Rev. Lett. 51, 71 (1983).

[12] The Darwin Correspondence project http:// www.darwinproject.ac.uk

[13] A. Vazquez, Phys. Rev. Lett. 95, 248701 (2005).

[14] A. Gabrielli and G. Caldarelli, Phys. Rev. Lett. 98, 208701
(2007).

[15] M. Marsili, J. Stat. Phys. 77, 733 (1994); A. Gabrielli, M. Marsili, R. Cafiero, and L. Pietronero, ibid. 84, 889 (1996).

[16] R. Cafiero, A. Gabrielli, M. Marsili, and L. Pietronero, Phys. Rev. E 54, 1406 (1996).

[17] A. Gabrielli, G. Caldarelli, and L. Pietronero, Phys. Rev. E 62, 7638 (2000).

[18] G. Grinstein and R. Linsker, Phys. Rev. Lett. 97, 130201 (2006).

[19] M. Paczuski, S. Maslov, and P. Bak, Phys. Rev. E 53, 414 (1996).

[20] S. Redner, A Guide to First-Passage Processes (Cambridge University, Cambridge, England, 2001).

[21] D. Stauffer and A. Aharony, Introduction to Percolation Theory (Taylor \& Francis, London, 1991).

[22] G. Grinstein and R. Linsker, Phys. Rev. E 77, 012101 (2008).

[23] N. Masuda, J. S. Kim, and B. Kahng, Phys. Rev. E 79, 036106 (2009).

[24] O. Angel, J. Goodman, F. den Hollander, and G. Slade, Ann. Probab. 36, 420 (2008). 